\title{
Trends in der Gesundheitswirtschaft
}

Überdurchschnittliche Wachstumsraten, starker Job-Zuwachs und hohe technische Innovationskraft: Die blanken Zahlen zeigen die Gesundheitswirtschaft als Wachstumstreiber und Jobmotor. Und doch steht die Branche in der Kritik, Gesundheit sei zu teuer, das System ineffizient und neue Behandlungsmethoden kommen nicht beim Patienten an. Ein kritischer Blick auf die heutigen Strukturen zeigt, welche Faktoren über den Erfolg und die Zukunft der Branche entscheiden werden.

Die durchschnittliche Wachstumsrate der Gesundheitswirtschaft mit 3,8 Prozent in den Jahren 2006 bis 2017 ist höher als vergleichbar große Wirtschaftszweige. Selbst der Dienstleistungssektor kann mit seinen 2,7 Prozent Wachstum hier nicht mithalten. Diese Entwicklung schreitet weiter voran: Das Bundesministerium für Wirtschaft (BMWi) teilt mit, dass die Bruttowertschöpfung um 12,8 Milliarden Euro gegenüber dem Jahr 2016 gestiegen ist. Jeder achte Euro wird für Gesundheit ausgegeben. Seit 2006 hat die Gesundheitswirtschaft insgesamt mehr als 1,4 Millionen Arbeitsplätze geschaffen und beschäftigt mit einem Anteil von rund 16,6 Prozent am Arbeitsmarkt der Gesamtwirtschaft fast so viele Erwerbstätige wie das verarbeitende Gewerbe. Nicht zu Unrecht wird die Branche deshalb häufig als Jobmotor bezeichnet.

Der Ökonom und Zukunftsforscher Leo A. Nefiodow hat bereits vor mehr als einem Jahrzehnt die Hypothese beschrieben, dass die Gesundheitswirtschaft im 21. Jahrhundert eine ökonomische Leitfunktion und damit die Stellung als Wachstumsmotor einnehmen wird (Kondratjew-Zyklen). Trotzdem ist dieser Wirtschaftszweig vielfach in der Kritik und das Wachstum erzeugt nicht nur Beifall.

Strukturschwächen und gesetzliche Rahmenbedingungen

Den kontinuierlich weiter steigenden Gesundheitsausgaben liegen vielfältige Ursachen zugrunde. Eine im internationalen Vergleich überdurchschnittliche Krankenhaus- und Ärztedichte, vorhandene

\section{Die Innovationskraft der medizinisch- technischen Indus- trie ist enorm. Seit Jahren führen solche Unternehmen, auch aus Deutschland, das Ranking der Patentanmeldungen beim Europäischen Patentamt an.}

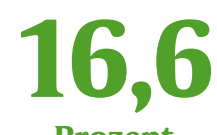

Prozent

der Erwerbstätigen in Deutschland werden von der Gesundheitswirtschaft beschäftigt. Damit ist ihr Anteil fast so hoch wie bei den Erwerbstätigen im verarbeitenden Gewerbe.

Doppelstrukturen in zwei Sektoren, Kommunikationsdefizite durch fehlende Digitalisierung, zunehmender Wettbewerb der Leistungserbringer, Fehlanreize im Vergütungssystem und auch der medizintechnische Fortschritt zählen zu den angebotsseitigen Einflussfaktoren.

Nachfragerseitig sind Krankenstand und die Arten der Erkrankungen innerhalb der Bevölkerung, das wenig vorhandene Kostenbewusstsein (für GKV-Versicherte nicht transparent), das gestiegene Infor- mations- und Anspruchsniveau der Menschen - jede diagnostizierte Erkrankung muss auch therapierbar sein - und nicht zuletzt auch die demografische Entwicklung als wesentliche Aspekte zu nennen.

Eine bundesweite Krankenhausbedarfsplanung scheitert am Föderalismus, der Leistungskatalog der gesetzlichen Krankenversicherung wird nicht mehr diskutiert, die Honorierung von stationärer Leistung soll zukünftig qualitätsorientiert erfolgen.

Laut Beratungsexperten ${ }^{1}$ trägt die angebotsinduzierte Nachfrage zu rekordverdächtigen Operationszahlen und Multimedikation bei, bedingt durch die sektorale Trennung und mangelhafte Koordination. Generell gelten der medizinisch-technische Fortschritt und die demografische Entwicklung als die beiden wesentlichen Treiber, die den Schereneffekt zwischen Einnahmen und Ausgaben am meisten beeinflussen. Grund dafür ist, dass sich das medizinische Wissen in weniger als fünf Jahren verdoppelt. Der medizinisch-technische Fortschritt eröffnet neue Möglichkeiten, neue diagnostische Verfahren ziehen oftmals innovative Therapieverfahren nach sich. So haben minimalinvasive Operationsmethoden, zum Beispiel die kathetergestützte Implantation von Herzklappen, dazu geführt, dass auch ältere Menschen von einem solchen Eingriff profitieren können und viele Jahre Lebensqualität gewinnen. Gleichzeitig konnten Indikationsstellungen erweitert werden, so dass die Zahl der operativen Eingriffe erheblich zugenommen hat. 
Trotz der Herausforderung, die in der beschriebenen Entwicklung liegt, ist dieser Behandlungsfortschritt natürlich zu begrüßen, denn die Gesundheitsversorgung hat sich dadurch verbessert und der volkswirtschaftliche Nutzen solcher modernen, patientenschonenden Therapien führt zu mehr Wachstum in unserer Gesellschaft. Nicht zu vergessen ist dabei, dass sie für viele Patienten auch weniger Schmerzen und schnellere Rekonvaleszenz bedeuten.

Die Innovationskraft der medizinisch-technischen Industrie ist enorm. Seit Jahren führen solche Unternehmen, auch aus Deutschland, das Ranking der Patentanmeldungen beim Europäischen Patentamt an - vor anderen innovativen Branchen wie Informationstechnologie, Elektronik etc. Der Bundesverband Medizintechnologie e. V. (BVMed) nennt in seinem Jahresbericht 2017/2018 ein Umsatzwachstum in Deutschland von 2,8 Prozent und beklagt gleichzeitig die fehlende Dynamik der Erstattungs- und Bewertungssysteme. Hier gilt es, die Entwicklung genau zu beobachten, um letztlich zu verhindern, dass innovative Produkte und Verfahren am Patienten in Deutschland vorbeigehen.

Wir dürfen also auch in Zukunft mit Leistungsausweitungen rechnen, so lautet das Fazit zu der dargestellten Situation. Leider steht dem - in steigendem Ausmaß - ein Mangel an Personalressourcen gegenüber. Sowohl in der stationären Versorgung als auch im Hausarztbereich fehlen Mediziner; noch dramatischer stellt sich die Situation bei Pflegekräften dar. Nur mit einer deutlichen Steigerung der Attraktivität für die Profession Pflege können die Anforderungen der Zukunft bewältigt werden.

Vorgenannte Ausführungen machen deutlich, dass alle Akteure innerhalb der Gesundheitswirtschaft gut beraten sind, die endlichen finanziellen Ressourcen angemessen und am Patienten orientiert einzusetzen. Eine ausgewogene Betrachtung von Qualität, Patientensicherheit und Wirtschaftlichkeit sollte dafür die Grundlage sein. Dies gilt insbesondere für die Beschaffung von Sachmitteln.

\section{Herausforderungen und Chancen für Gesundheitseinrichtungen}

Am Beispiel der stationären Versorgung lassen sich Chancen und Herausforderungen transparent machen. Die Krankenhäuser sehen sich seit Jahren einem Mehr an Wettbewerb gegenüber, sie konkurrieren in mehreren Teilmärkten der Gesundheitswirtschaft.

Ihre Zukunftsfähigkeit hängt entscheidend an den Erfolgsfaktoren „bestmögliche Qualität bei der Leistungserbringung “ und „wirtschaftliche Rahmenbedingungen“. Eine stringente Kosten- und Erlöskontrolle ist zwingend notwendig. Das Leistungsspektrum ist den regionalen Gegebenheiten, dem eigenen Leistungsvermögen und nicht zuletzt den gesetzlichen Vorgaben anzupassen (Stichwort „Mindestmengen“). Die Gesamtkosten, Personal- und Sachkosten, steigen weiter. Professionelles Personalmanagement, Personalentwicklung vs. Personalverwaltung, Employer Branding etc. sind wesentliche kritische Erfolgsfaktoren. Der Wille zur Innovation und die Investitionskraft sichern neben intelligentem Management die Zukunft. Es gilt, Standards in medizinischen und administrativen Bereichen einzuführen, ohne die Individualität der Patienten in

\section{BUCH TIPP}
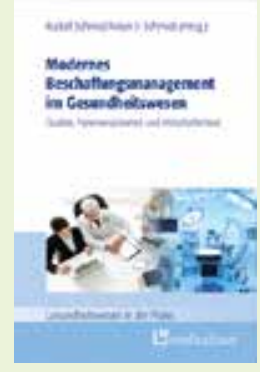

Modernes Beschaffungsmanagement im Gesundheitswesen,

Rudolf Schmid, Anton J. Schmidt (Hrsg), medhochzwei Verlag; 2018
Gänze außer Acht zu lassen. Vorhandene Kostensenkungspotenziale, zum Beispiel beim Personaleinsatz oder der Beschaffung, sind ganzheitlich darzustellen und zwingend zu realisieren. Effektivität und Effizienz der medizinischen Kernprozesse sind bspw. durch die Einführung von intelligenten klinischen Behandlungspfaden zu verbessern.

Sekundäre und tertiäre Leistungsbereiche, die keinen oder nur geringe Wertbeiträge am Patienten liefern, sollten hinterfragt und gegebenenfalls mit einem externen System- oder besser mit einem Prozesspartner erbracht werden.

Eine große Chance liegt in der verbesserten Zusammenarbeit der Berufsgruppen Medizin, Pflege und Administration im Krankenhaus, besonders auch im Bereich Beschaffung. Wünschenswert wäre es, dass die heute noch oftmals vorhandene abteilungsbezogene, häufig voneinander unabhängige Leistungserbringer-Struktur von einer integrierten, voneinander abhängigen und koordinierten Organisationsstruktur ersetzt wird. Dies würde bedeuten, die prozessuale Betrachtung löst die funktionale Betrachtung ab, die Leistung orientiert sich am Patienten.

Hier ist insbesondere auch professionelles Beschaffungsmanagement gefordert. Das „richtige“ Produkt für den „richtigen“ Patienten kann nur berufsgruppenübergreifend (Anwender und Ökonom) definiert werde. Beschaffungsmanagement ist deshalb „Mannschaftssport“.

\section{Literatur:}

${ }^{1}$ Niestroj, B./Matusiewicz, D.: Die Megawelle Gesundheit - über den Wachstumsmotor des 21. Jahrhunderts. In: Health \& Care Management 9/2017, S. 56-57 